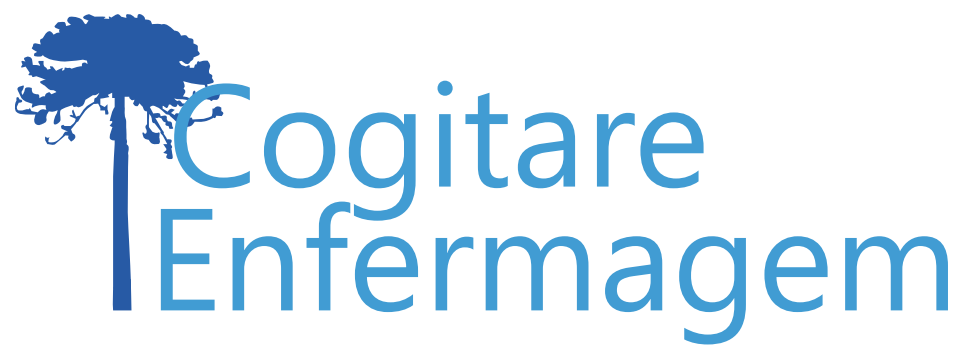

\title{
INTERVENÇÃO DA ENFERMAGEM NA PREVENÇÃO DE QUEDA EM IDOSO: ESTUDO DE REVISÃO
}

Bebiano Will dos Santos ${ }^{1}$, Cristina Lavareda Baixinho²

\section{RESUMO}

Objetivo: identificar as intervenções de enfermagem que aumentam a segurança da casa do idoso e previnem a queda.

Método: revisão integrativa da literatura orientada pela questão: 'Que intervenções de enfermagem são adequadas à prevenção de queda no domićlio da pessoa idosa?'. Entre maio e junho de 2018 identificaram-se estudos na EBSCO, B-On, SCOPUS, ISI e JBI.

Resultados: os 13 estudos recomendam intervenções que foram organizadas em cinco categorias: avaliação do risco ambiental; aconselhamento sobre modificações; adaptação do espaço e equipamentos; assessoria na aquisição de produtos de apoio; e estratégias cognitivocomportamentais que aumentam a segurança do idoso na utilização do espaço físico da casa. Conclusão: os achados desta revisão permitiram mapear as intervenções que podem ser introduzidas no domićlio do idoso para evitar a queda, a sua recorrência e as lesões associadas.

DESCRITORES: Acidentes por Quedas; Idoso; Habitação; Enfermagem; Segurança de Equipamentos.

COMO REFERENCIAR ESTE ARTIGO:

Santos BW dos, Baixinho CL. Possibilidade de intervenção da enfermagem na prevenção de queda em idoso: estudo de revisão. Cogitare enferm. [Internet]. 2020 [acesso em "colocar data de acesso, dia, mês abreviado e ano"]; 25. Disponível em: http://dx.doi.org/10.5380/ce.v25i0.71326.

${ }^{1}$ Enfermeiro. Mestre em Enfermagem de reabilitação. Enfermeiro na Unidade de saúde Familiar Rodrigues Migueis. Lisboa, Portugal. $\odot$

${ }^{2}$ Enfermeira. Doutora em Enfermagem. Docente do Departamento de Enfermagem de Reabilitação, Escola Superior de Enfermagem de Lisboa. Lisboa, Portugal. 


\title{
POSSIBILITIES FOR NURSING INTERVENTIONS IN THE PREVENTION OF FALLS IN THE ELDERLY: A REVIEW
}

\begin{abstract}
Objective: To identify the nursing interventions that increase the home safety of elderly and prevent falls.

Method: Integrative literature review guided by the question: "What nursing interventions are adequate to prevent falls in the elderly at home?". From May to June 2018, there was a search for studies in EBSCO, B-On, SCOPUS, ISI, and JBI.

Results: The 13 studies recommend interventions that were organized in five categories: environmental risk assessment; advices on changes; adaptation of the space and equipment; assistance in the purchasing of support products and cognitive-behavioral strategies that increase the safety of the elder in the use of the physical space at home.

Conclusion: The possible interventions for the prevention of falls include: environmental risk assessment; advices on changes; adaptation of the space and equipment; assistance in the purchasing of support products; and cognitive-behavioral strategies that increase the safety of the elder in the use of the physical space at home.
\end{abstract}

DESCRIPTORS: Accidental Falls; Aged; Housing; Nursing; Equipment Safety.

\section{POSIBILIDAD DE INTERVENCIÓN DE ENFERMERÍA EN LA PREVENCIOON DE LA CAÍDA EN PERSONAS MAYORES: ESTUDIO DE REVISIÓN}

\begin{abstract}
RESUMEN:
Objetivo: identificar intervenciones de enfermería que aumenten la seguridad del hogar de ancianos y eviten caídas.

Método: Revisión integral de la literatura guiada por la pregunta: "¿Qué intervenciones de enfermería son apropiadas" para evitar caídas en el hogar de la persona mayor? Entre mayo y junio de 2018, se identificaron estudios en EBSCO, B-On, SCOPUS, ISI y JBI.

Resultados: los 13 estudios recomiendan intervenciones que se organizaron en cinco categorías: evaluación de riesgos ambientales; asesoramiento sobre modificaciones; adaptación de espacio y equipamiento; Asesoramiento en la adquisición de productos de apoyo y estrategias cognitivo-conductuales que aumenten la seguridad de las personas mayores en el uso del espacio físico del hogar.

Conclusión: Las posibilidades de intervención en la prevención de caídas, van desde la evaluación del riesgo ambiental; asesoramiento sobre modificaciones y adaptación de espacio y equipamiento; Asesoramiento sobre la adquisición de productos y estrategias cognitivoconductuales que aumenten la seguridad de las personas mayores en el uso del espacio físico del hogar.
\end{abstract}

DESCRIPTORES: Accidentes por caídas; Anciano; Vivienda; Enfermería; Seguridad de Equipos. 
As quedas são a terceira causa de incapacidade crônica em idosos, constituindose numa síndrome geriátrica com impacto negativo na funcionalidade da pessoa idosa que cai e/ou tem medo de cair ${ }^{(1)}$. O aumento da esperança média de vida leva a que este evento adverso seja observado com interesse pela investigação e pelos profissionais da clínica, porque são uma "growth area", com impacto negativo na funcionalidade ${ }^{(2)}$, sendo responsáveis por uma elevada morbilidade, mortalidade, sofrimento e aumento do custo com o tratamento das lesões secundárias a este evento ${ }^{(2-4)}$.

Com uma prevalência de cerca de $30 \%(4-6)$, este problema agrava-se com o avançar da idade. Enquanto que no grupo etário dos 65 anos, um terço dos idosos cai, pelo menos uma vez por ano; acima dos 85 anos este valor aumenta para $50 \%(5)$, sendo que uma em cada cinco quedas exige cuidados de saúde, porque provoca lesões moderadas ou graves, tendo um em cada dez incidentes de queda como resultado uma fratura ${ }^{(2-4)}$.

Autores alertam que, no contexto comunitário, este evento adverso tem consequências nefastas, promovendo o aumento da dependência pela incapacidade funcional associada às lesões ou pelos efeitos que o medo de queda tem na pessoa idosa ${ }^{(4-7)}$, que impõe ou vê ser-lhe imposta um conjunto de restrições à atividade, que a remetem para um estado de passividade física, psicológica e social(2,4), preditor não só da perda da qualidade de vida, mas também de institucionalização ${ }^{(8,9)}$. Assim, as quedas, pela sua prevalência e impacto no indivíduo, família e sociedade, constituem uma preocupação para a saúde pública ${ }^{(9)}$.

A sua prevenção é difícil pela natureza multidimensional dos fatores de risco. Por isso, além da avaliação do risco e introdução das medidas preventivas, individualizadas a cada pessoa idosa, há que considerar fatores de risco biofisiológico, psicológicos, socioeconômicos e ambientais ${ }^{(2,10-13)}$.

As recomendações internacionais observam a importância das visitas preventivas para a manutenção da saúde e independência, que consequentemente previnem incapacidades e hospitalizações desnecessárias, reduzindo custos ${ }^{(4,14)}$. A visita atempada ao domicílio é, por si só, uma estratégia valiosíssima para a avaliação do risco de queda relacionado com fatores ambientais ${ }^{(15)}$, para a prevenção da queda nas pessoas mais velhas na comunidade ${ }^{(2,4)}$, não só pela intervenção imediata, mas também pelo planejamento de intervenções futuras, incluindo o envolvimento de outros profissionais ${ }^{(2,14)}$.

Todavia, as conclusões dos estudos não são claras quanto à eficácia de algumas intervenções efetuadas no domicílio acerca da redução do risco e prevalência de queda ${ }^{(16)}$. Logo, o objetivo do estudo foi identificar as intervenções de enfermagem que aumentam a segurança da casa do idoso e previnem a queda.

\section{MÉTODO}

A presente Revisão Integrativa da Literatura (RIL) foi orientada pela seguinte questão de investigação, elaborada de acordo com a mnemônica PICo (população, fenômeno de interesse e contexto): "Que intervenções de enfermagem são adequadas para a prevenção de queda no domicílio da pessoa idosa?".

Recorreu-se a um protocolo com seis etapas para operacionalizar a pesquisa: identificação do tema e seleção da hipótese ou questão de pesquisa; estabelecimento de critérios para inclusão e exclusão de estudos; definição das informações a serem extraídas; avaliação dos estudos incluídos; interpretação dos resultados; e apresentação da revisão/ síntese do conhecimento(17). 
Esta orientação metodológica possibilitou a definição dos critérios de elegibilidade dos estudos, com a finalidade de estreitar os intervalos de confiança, facilitar a comparação dos trabalhos, interpretar os dados e aumentar a precisão dos resultados. Os critérios de inclusão previamente definidos foram: $(P)$ - artigos cuja população ou amostra fossem pessoas idosas ( $\geq 65$ anos); (I) - estudos sobre as intervenções no espaço físico da casa (para aumentar a segurança, melhorar a acessibilidade, eliminar riscos) que tenham impacto positivo no risco de cair, na queda e/ou nas lesões resultantes e, por último, artigos cujo contexto (Co) se reporta à casa da pessoa. Excluíram-se os artigos cuja população eram adultos ou idosos hospitalizados ou institucionalizados.

Os descritores MeSH utilizados na pesquisa, efetuada entre os meses de maio a junho de 2018, foram: ("interventions" or "prevention") and ("community" or "home visit" or "home hazard modification" or "home modifications") and ("elderly" or "aging") and ("falls" or "accidental falls") and ("architectural accessibility" or "safety" or "security measures" or "equipment safety" or "patient safety").

A busca foi efetuada em três idiomas (português, espanhol e inglês). Recorreu-se às bases de dados disponíveis nos motores de busca da EBSCO, B-On, SCOPUS, ISI (Web of Sience) e JBI (Joanna Briggs Institute). Pela atualidade do tema e face ao elevado número de estudos realizados nos últimos anos, optou-se por colocar como limite temporal para os artigos da amostra: 2013 a 2018.

Os investigadores construíram uma tabela Excel para registarem o conteúdo extraído dos artigos da amostra bibliográfica final: identificação do título do artigo/obra; autor(es), ano de publicação, tipo de artigo; objetivo(s), método e técnicas; nível de evidência; e principais resultados/conclusões.

A partir das recomendações PRISMA, identificaram-se 39 artigos com potencialidade para responder à questão de investigação e que respeitavam os critérios de elegibilidade. A leitura do título possibilitou a identificação de 3 artigos repetidos. A leitura do resumo permitiu excluir 10 estudos que não respeitavam os critérios de inclusão. Leu-se na íntegra 26 artigos, dos quais metade foi excluída por não responder à questão de investigação. Os 13 artigos finais constituem a amostra bibliográfica final.

Os resultados dos artigos que permitiam responder à questão de investigação foram extraídos e submetidos à análise de conteúdo. Na definição das categorias, garantiu-se a representatividade, a exaustividade, a homogeneidade e a pertinência das mesmas para o objeto de estudo. Dois investigadores fizeram a codificação, que foi posteriormente aferida pelos outros para aumentar a fidedignidade da análise.

\section{RESULTADOS}

Os 13 estudos da amostra bibliográfica (Quadro 1) não são homogêneos, tendo objetivos e desenhos de estudo diferentes, dificultando a comparação dos resultados. Todavia, embora registem diferenças das fontes e da metodologia, a sua interpretação permite dar resposta à questão de investigação.

Quadro 1 - Estudos constituintes da amostra bibliográfica, Lisboa, Portugal, 2018 (continua)

\begin{tabular}{|l|l|l|}
\hline Autores (ano) & Tipo de Estudo & Principais Resultados \\
\hline $\begin{array}{l}\text { Oliveira, Baixinho } \\
\text { e Henriques } \\
(2018)^{(4)}\end{array}$ & Scoping review & $\begin{array}{l}\text { Os resultados apontam para que o reforço comportamental } \\
\text { associado à assessoria na modificação ambiental diminua a } \\
\text { prevalência de quedas. }\end{array}$ \\
\hline
\end{tabular}




\begin{tabular}{|c|c|c|}
\hline Oxtoby $(2017)^{(18)}$ & $\begin{array}{l}\text { Revisão da } \\
\text { literatura }\end{array}$ & $\begin{array}{l}\text { A avaliação no domicílio do idoso possibilita a identificação } \\
\text { dos fatores de risco de queda e a implementação de medidas } \\
\text { preventivas não só para o espaço físico da casa, mas também } \\
\text { para o idoso, por exemplo, uso de calçado adequado à marcha, } \\
\text { existência de fios ou tapetes soltos, iluminação inadequada, } \\
\text { especialmente em corredores e escadas. }\end{array}$ \\
\hline $\begin{array}{l}\text { Cabrita e José } \\
(2013)^{(19)}\end{array}$ & $\begin{array}{l}\text { Estudo } \\
\text { descritivo, } \\
\text { transversal, } \\
\text { quantitativo }\end{array}$ & $\begin{array}{l}\text { Os autores recomendam o controle dos riscos ambientais através } \\
\text { da gestão de risco e promoção da segurança do domicílio e } \\
\text { adoção de comportamentos seguros. }\end{array}$ \\
\hline $\begin{array}{l}\text { Palvanen et al. } \\
(2014)^{(20)}\end{array}$ & $\mathrm{RCT}$ & $\begin{array}{l}\text { A introdução de um programa com intervenções múltiplas que } \\
\text { integrou a modificação ambiental foi eficaz na diminuição do risco, } \\
\text { na prevalência de quedas e das lesões relacionadas em cerca de } \\
30 \% \text {. }\end{array}$ \\
\hline $\begin{array}{l}\text { Avin et al. (2015) } \\
\text { (9) }\end{array}$ & $\begin{array}{l}\text { Revisão } \\
\text { Sistemática }\end{array}$ & $\begin{array}{l}\text { A triagem de fatores de risco e a prescrição de intervenções } \\
\text { adaptadas de avaliação ambiental podem diminuir a prevalência } \\
\text { dos incidentes de queda. }\end{array}$ \\
\hline $\begin{array}{l}\text { Kamei et al. } \\
(2015)^{(21)}\end{array}$ & RCT & $\begin{array}{l}\text { A informação sobre os fatores de risco ambiental e } \\
\text { comportamentos de segurança relacionados com a modificação } \\
\text { ambiental levaram à diminuição da prevalência em } 12 \text { semanas, } \\
\text { nos idosos com } 75 \text { anos, em } 18,5 \% \text {. }\end{array}$ \\
\hline $\begin{array}{l}\text { Maggi et al. } \\
(2018)^{(22)}\end{array}$ & $\begin{array}{l}\text { Quasi- } \\
\text { experimental }\end{array}$ & $\begin{array}{l}\text { Os resultados demonstram que as alterações no ambiente da casa, } \\
\text { com modificação dos fatores de risco, têm impacto na melhoria } \\
\text { da qualidade de vida da pessoa idosa, bem como para sua } \\
\text { manutenção no domicílio por mais tempo. }\end{array}$ \\
\hline $\begin{array}{l}\text { Wilson, } \\
\text { Kvizhinadze, } \\
\text { Pega, Nair e } \\
\text { Blakely }(2017)^{(23)} \\
\end{array}$ & $\begin{array}{l}\text { Estudo de } \\
\text { modelagem }\end{array}$ & $\begin{array}{l}\text { A intervenção, que incluía a avaliação da segurança conjugada } \\
\text { com modificações ambientais, aponta para uma redução do risco } \\
\text { e da prevalência da queda, com ganhos consideráveis de saúde, } \\
\text { sendo custo-efetiva. }\end{array}$ \\
\hline $\begin{array}{l}\text { Pega, } \\
\text { Kvizhinadze, } \\
\text { Blakely, Atkinson } \\
\text { e Wilson }(2016)^{(24)}\end{array}$ & $\begin{array}{l}\text { Estudo de } \\
\text { modelagem }\end{array}$ & $\begin{array}{l}\text { Os resultados da intervenção, que incluiu avaliação da segurança } \\
\text { e modificações ambientais na casa do idoso, provam que há } \\
\text { vantagens econômicas. }\end{array}$ \\
\hline $\begin{array}{l}\text { Ueda et al. (2017) } \\
\text { (25) }\end{array}$ & $\begin{array}{l}\text { Estudo } \\
\text { randomizado }\end{array}$ & $\begin{array}{l}\text { O grupo de intervenção, com intervenção no espaço físico e } \\
\text { acessibilidades, teve } 75 \% \text { menos quase-quedas em comparação } \\
\text { com o grupo controle (hazard ratio, } 0.25 ; 95 \% \text { intervalo de } \\
\text { confiança, } 0.09-0.75) \text {. }\end{array}$ \\
\hline $\begin{array}{l}\text { Rimland et al. } \\
(2017)^{(26)}\end{array}$ & $\begin{array}{l}\text { Estudo } \\
\text { metodológico, } \\
\text { qualitativo } \\
\text { (Painel de } \\
\text { peritos) }\end{array}$ & $\begin{array}{l}\text { Aos idosos residentes na comunidade, com alto risco de cair, é } \\
\text { recomendada a avaliação de segurança em casa e modificação } \\
\text { para reduzir a prevalência (forte recomendação, evidência de } \\
\text { qualidade moderada). }\end{array}$ \\
\hline $\begin{array}{l}\text { Stark et al. } \\
(2018)^{(27)}\end{array}$ & $\begin{array}{l}\text { Estudo } \\
\text { randomizado }\end{array}$ & $\begin{array}{l}\text { Os investigadores recomendam a modificação domiciliária por ser } \\
\text { viável nos idosos com vulnerabilidade acrescida na comunidade. }\end{array}$ \\
\hline $\begin{array}{l}\text { Rimland et al. } \\
(2016)^{(28)}\end{array}$ & $\begin{array}{l}\text { Revisão } \\
\text { Sistemática }\end{array}$ & $\begin{array}{l}\text { A intervenção ambiental deve constar como medida preventiva de } \\
\text { quedas na população idosa residente na comunidade e deve fazer } \\
\text { parte desta a avaliação da segurança e dos riscos de casa, bem } \\
\text { como a realização de modificações. }\end{array}$ \\
\hline
\end{tabular}

Fonte: Os autores (2018).

Uma análise detalhada dos resultados dos estudos permitiu constatar que era possível organizar os resultados em cinco categorias de ações relacionadas com a intervenção dos 
profissionais de saúde. As categorias que emergiram foram: avaliação do risco ambiental; aconselhamento sobre modificações ambientais; adaptação do espaço e equipamentos; assessoria na aquisição de produtos de apoio que aumentem a segurança do ambiente; e estratégias cognitivo-comportamentais que aumentam a segurança do idoso na utilização do espaço físico da casa.

\section{DISCUSSÃO}

Os 13 estudos que integram a amostra final obedecem aos critérios de inclusão e exclusão previamente definidos e possibilitam responder à questão e ao objetivo desta RIL. Os estudos incluídos são predominantemente primários $(n=9)^{(19-27)}$ e de natureza quantitativa $(n=8)^{(19-25,27)}$, com quatro $\operatorname{RCT}^{(20,21,25,27)}$, dois estudos de modelagem ${ }^{(23,24)}$, um estudo quasi-experimental(22), um estudo descritivo(19), quatro estudos são revisões de literatura com metodologia científica ${ }^{(4,9,18,28)}$ e um estudo é qualitativo e metodológico (painel de peritos) ${ }^{(26)}$.

Os estudos são díspares em termos de método e técnicas, utilizando instrumentos diversificados para a avaliação do risco e das lesões associadas. As amostras têm tamanhos diferentes e a intervenção implementada, apesar de introduzir alterações na segurança da casa, é apresentada isoladamente ou conjugada com outras intervenções que diferem de estudo para estudo. Os momentos de avaliação da efetividade da intervenção também são diferentes, o que dificulta a comparação dos resultados.

Nos estudos de natureza quantitativa, as médias de idades dos idosos são diferentes, bem como os critérios de inclusão relativos a esta variável. Por exemplo, num dos estudos, os 1314 idosos que participaram tinham idade igual ou superior a 70 anos ${ }^{(20)}$, o que pode alterar os resultados e dificulta comparações, porque o acréscimo de anos está relacionado com o aumento do risco, da prevalência e das lesões associadas à queda, sendo que a maior prevalência de risco de queda $(50,5 \%)$ é encontrada nos idosos com idade $\geq 80$ anos $^{(29)}$.

Em outro estudo da amostra, os participantes têm idades compreendidas entre os 65 anos e os 92 anos $^{(4)}$. A diferença de 27 anos têm traduções no risco, prevalência e na gravidade das lesões associadas à queda ${ }^{(2,30)}$ e influencia a percepção única da experiência de envelhecimento e da probabilidade da existência, ou não, de declínio funcional e cognitivo e até do medo associado à história anterior de algum evento adverso ${ }^{(2,4)}$.

Concordamos com os autores que advogam que idades diferentes requerem uma abordagem diferenciada para a prevenção da queda e da sua recorrência, para a gestão do risco ambiental, bem como para a implementação de intervenções que devem respeitar a heterogeneidade deste grupo populacional ${ }^{(2,4)}$.

Nos estudos da amostra, não fica claro se a intervenção foi sempre aplicada pelo mesmo enfermeiro ou pela mesma equipe de saúde, não é igualmente perceptível se as equipes tiveram formação sobre a gestão do risco de queda. Os estudos apontam que

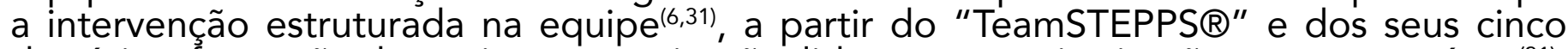
domínios: formação da equipe, comunicação, liderança, monitorização e suporte mútuo(31), pode melhorar a intervenção junto dos idosos com risco de queda.

Na categoria avaliação do risco ambiental, emerge a recomendação, consensual em todos os estudos, para a avaliação de segurança dentro de portas, de modo a identificar os riscos presentes nas casa do idosos, sobretudo nos que têm alto risco de queda ${ }^{(2,4,26)}$. A adequação da avaliação do risco conjugada com a implementação das medidas preventivas que o controlam ${ }^{(15)}$ traduzem-se em vantagens econômicas ${ }^{(24)}$.

A visita domiciliária, para esse efeito, pode, por si, ajudar a identificar e prevenir os riscos de queda ${ }^{(2,4,9,18)}$, possibilitando a individualização das intervenções a partir dos riscos 
reais avaliados em contexto ${ }^{(9,18)}$, permitindo a intervenção imediata e o planejamento de intervenções a médio e longo prazo, incluindo o envolvimento de outros profissionais ${ }^{(15)}$, motivando o idoso, aumentando a adesão ao regime terapêutico, à segurança e a satisfação do cliente ${ }^{(2,14)}$.

A visita domiciliária preventiva, com objetivos bem definidos, pode fortalecer a autoestima, promover o controle sobre o risco e sobre a tomada de decisão na implementação de medidas preventivas, e torna os idosos mais conscientes da importância da prevenção para se manterem ativos durante mais tempo ${ }^{(14)}$.

Em relação à categoria aconselhamento sobre as modificações ambientais, os achados demonstram que há evidência de que quedas podem ser evitadas através de alterações simples ${ }^{(2,4,9)}$, como remover ou fixar os tapetes soltos, a avaliação de pés e calçado e seleção de calçado adequado às características do pé e da marcha ${ }^{(4,9)}$. A assessoria na modificação ambiental e o reforço comportamental diminuem a prevalência de quedas ${ }^{(12)}$ e aumentam a mobilidade e a segurança, quando são eliminados obstáculos nas zonas de passagem ${ }^{(11)}$. Os resultados da pesquisa apontam que estas intervenções são prioritárias nas pessoas com história de queda anterior e/ou alto risco de queda ${ }^{(9)}$.

A utilização de maquetes para informar e assessorar os idosos sobre a segurança habitacional diminui a prevalência de quedas nas pessoas com idade igual ou superior a 75 anos $^{(21)}$. Estudos futuros deverão explorar o impacto de outras estratégias, nomeadamente as novas tecnologias, como um recurso para a clínica, possibilitando uma melhor gestão dos recursos e disseminando a evidência ${ }^{(2)}$.

$\mathrm{Na}$ categoria a respeito da adaptação de espaço e equipamentos, incluem-se as intervenções efetivas que introduzem mudanças no espaço da casa e que demonstram ser eficazes na redução do risco, da prevalência e das lesões secundárias a este acidente em cerca de $30 \%{ }^{(20)}$.

O impacto econômico destas intervenções deve ser mensurado, apesar de algumas modificações não implicarem gastos elevados, como retirar tapetes, afastar mobiliário que dificulta a mobilidade dentro de casa, retirar fios soltos, entre outros ${ }^{(18,28)}$. Outras poderão envolver custos com a aquisição de produtos de apoio para a realização das atividades de vida diária e que o idoso e a sua família podem não conseguir comportar.

Os estudos de impacto econômico sobre o fenômeno em estudo apontam que esta intervenção pode produzir ganhos consideráveis de saúde e ser custo-efetiva. Assim, os investigadores recomendam a sua implementação nas pessoas idosas com quedas anteriores $^{(23,24)}$, tendo em conta que os resultados da investigação demonstram que as intervenções diminuem a prevalência, o risco e melhoram o desempenho no autocuidado(27), que é um fator de risco modificável.

$\mathrm{Na}$ categoria assessoria para a aquisição de produtos de apoio que aumentem a segurança do ambiente, os investigadores recomendam a avaliação da necessidade, aquisição, apoio no treino e supervisão na utilização de: iluminação adequada dos espaços; soluções antiderrapantes para pavimentos, banheira e chuveiro; barras de apoio lateral para facilitar a independência no autocuidado (eliminação, banho e arranjo pessoal); assento elevado de vaso sanitário que facilite o sentar e levantar; corrimãos nos corredores e nas escadas para facilitar o apoio na marcha e no subir e descer as escadas, entre outros ${ }^{(2,28)}$.

Em grupos com condições de saúde específicas, por exemplo, ter sofrido queda anterior com fratura, a introdução de uma intervenção singular como a modificação de pavimento foi importante para reduzir as quedas nos idosos após alta hospitalar de um serviço ortopédico, com o grupo de intervenção a ter $75 \%$ menos quase-quedas em comparação com o grupo controle ${ }^{(25)}$.

Em relação às estratégias cognitivo-comportamentais para promoção da adesão a comportamentos seguros na mobilidade e na utilização do espaço e equipamentos da casa, os achados demonstram que estas têm um efeito positivo na redução do risco, da 
prevalência e da morbilidade associada à lesão, onde se inclui o declínio cognitivo e físico, mortalidade e risco de institucionalização(4).

Num dos estudos incluídos para análise nesta RIL, o recurso a estratégias cognitivocomportamentais provou ter efeitos positivos na redução da restrição da atividade associada ao medo de cair(13). Este dado precisa ser explorado em investigações futuras, porque o medo de cair tem traduções graves na funcionalidade da pessoa idosa, quer por falta de confiança na realização das atividades de vida diária, quer na restrição de atividade e menor participação social| ${ }^{3,6}$.

As limitações do estudo são decorrentes da heterogeneidade da amostra, dos métodos, técnicas e instrumentos utilizados nos diferentes estudos. A pesquisa incluiu estudos em português, espanhol e inglês, não identificando estudos publicados em outras línguas e não tendo sido incluída literatura cinzenta, pelo que é possível que alguns estudos de mestrado e doutoramento com avaliação de intervenções na casa do idoso tenham se perdido.

\section{CONSIDERAÇÕES FINAIS}

Os achados desta revisão permitiram mapear as intervenções que devem ser introduzidas no domicílio do idoso para evitar a queda, a sua recorrência e as lesões associadas.

As intervenções identificadas foram organizadas em cinco categorias: avaliação do risco ambiental; aconselhamento sobre modificações ambientais; adaptação do espaço e equipamentos; assessoria na aquisição de produtos de apoio que aumentem a segurança do ambiente e estratégias cognitivo-comportamentais que aumentam a segurança do idoso na utilização do espaço físico da casa.

\section{REFERÊNCIAS}

1. Walker GM, Armstrong S, Gordon AL, Robertson K, Ward M, Conroy S, et al. The Falls In Care Home study: a feasibility randomized controlled trial of the use of a risk assessment and decision support tool to prevent falls in care homes. Clínic Reab. [Internet]. 2016 [acesso em 23 jan 2019]; 30(10). Disponível em: https://doi.org/10.1177/0269215515604672.

2. Santos BW, Baixinho CL. Intervenções de enfermagem no espaço físico da casa para prevenir a queda no idoso: Revisão Integrativa da Literatura. In: Costa AP, Sá AA., Castro P, Souza DN. Atas do $8^{\circ}$ Congresso lbero-Americano em Investigação Qualitativa. 2019. Oliveira de Azeméis: Ludomedia, pp. 91-100. Disponível em: https://proceedings.ciaiq.org/index.php/CIAIQ2019/issue/archive.

3. Houry D, Florence C, Baldwin G, Stevens J, McClure R. The CDC Injury Center's response to the growing public health problem of falls among older adults. Am J Lifestyle Med. [Internet]. 2015 [acesso em 23 jan 2019]; 10(1). Disponível em: http://dx.doi.org/10.1177/1559827615600137.

4. Oliveira T, Baixinho CL, Henriques A. Prevention of Falls - Interventions in the Home Visits to the Elderly: Scoping Review. IJOCS. [Internet]. 2018 [acesso em 02 fev 2019]; 12(1). Disponível em: https:// www.researchgate.net/publication/325448376 Prevention of Falls - Interventions in the Home Visits to the Elderly Scoping Review.

5. Buckinx F, Rolland Y, Reginster JY, Ricour C, Petermans J, Bruyère O. Burden of frailty in the elderly population: perspectives for a public health challenge. Arch Public Health. [Internet]. 2015 [acesso em 23 nov 2019]; 73(19). Disponível em: http://dx.doi.org/10.1186/s13690-015-0068-x. 
6. Baixinho $C L$, Dixe $M$ dos $A$. Práticas das equipas na prevenção de queda nos idosos institucionalizados: construção e validação de escala. Texto contexto - enferm. [Internet]. 2017 [acesso em 11 fev 2019]; 26(3). Disponível em: http://dx.doi.org/10.1590/0104-07072017002310016.

7. Mitchell SE, Aitken SA, Court-Brown CM. The Epidemiology of Fractures Caused by Falls Down Stairs. ISRN Epidemiology. [Internet]. 2013 [acesso em 02 fev 2019]; ID370340. Disponível em: http://dx.doi. org/10.5402/2013/370340.

8. Rodríguez-Molinero A, Narvaiza L, Gálvez-Barrón S, Cruz JJ de la, Ruíz J, Gonzalo N, et al. Falls in the Spanish elderly population: Incidence, consequences and risk factors. Rev Esp Geriatr Gerontol. [Internet]. 2015 [acesso em 02 fev 2019]; 50(6). Disponível em: http://dx.doi.org/10.1016/j.regg.2015.05.005.

9. Avin KG, Hanke TA, Kirk-Sanchez N, McDonough CM, Shubert TE, Hardage J, Hartley G. Management of Falls in Community- Dwelling Older Adults: Clinical Guidance Statement From the Academy of Geriatric Physical Therapy of the American Physical Therapy Association. Phys Ther, [Internet]. 2015 [acesso em 18 jun 2018]; 95(6). Disponível em: http://dx.doi.org/10.2522/ptj.20140415.

10. Kim EJ, Arai H, Chan P, Chen LK, Hill KD, Kong B, et al. Strategies on fall prevention for older people living in the community: A report from a round-table meeting in IAGG 2013. J. Clinical Gerontology abd Geriatrics. [Internet]. 2015 [acesso em 17 ju 2018]; 6(2). Disponível em: https://doi.org/10.1016/j. jcgg.2015.02.004.

11. Sherrington C, Michaleff ZA, Fairhall N, Paul SS, Tiedemann A, Whitney J, et al. Exercise to prevent falls in older adults: an updated systematic review and meta-analysis. Br J Sports Med. [Internet]. 2016 [acesso em 17 jun 2018]; 51(24). Disponível em: http://dx.doi.org/10.1136/bjsports-2016-096547.

12. Luck T, Motzek T, Luppa M, Matschienger H, Fleischer S, Sesselmann Y, et al. Effectiveness of preventive home visits in reducing the risk of falls in old age: a randomized controlled trial. Clin Interv Aging. [Internet]. 2013 [acesso em 18 jun 2018]; 8. Disponível em: https://doi.org/10.2147/CIA.S43284.

13. Dorresteijn TAC, Zijlstra GAR, Ambergen AW, Delbaere K, Vlaeyen JWS, Kempen GIJM. Effectiveness of a home-based cognitive behavioral program to manage concerns about falls in community-dwelling, frail older people: results of a randomized controlled trial. BMC Geriatrics. [Internet]. 2016 [acesso em 15 jun 2018]; 16(2). Disponível em: https://doi.org/10.1186/s12877-015-0177-y.

14. Behm L, Ivanoff SD, Zidén L. Preventive home visits and health - experiences among very old people. BMC Public Health [Internet]. 2013 [acesso em 18 jun 2018]; 13(378). Disponível em: https://doi. org/10.1186/1471-2458-13-378.

15. Grant S, Parsons A, Burton J, Montgomery P, Underhill K, Mayo-Wilson E. Home Visits for Prevention of Impairment and Death in Older Adults: A Systematic Review. Campbell Systematic Reviews [Internet]. 2014 [acesso em 17 jun 2018]; 10(1). Disponível em: https://doi.org/10.4073/csr.2014.3.

16. Mayo-Wilson E, Grant S, Burton J, Parsons A, Underhill K, et al. Preventive Home Visits for Mortality, Morbidity, and Institutionalization in Older Adults: A Systematic Review and Meta-Analysis. PLoS ONE [Internet]. 2014 [acesso em 16 jun 2018]; 9(3). Disponível em: https://doi.org/10.1371/journal. pone.0089257.

17. Mendes KDS, Silveira RC de CP, Galvão CM. Revisão integrativa: método de pesquisa para a incorporação de evidências na saúde e na enfermagem. Texto contexto-enferm. [Internet]. 2008 [acesso em 20 fev 2018]; 17(4). Disponível em: http://dx.doi.org/10.1590/S0104-07072008000400018.

18. Oxtoby K. Preventing falls in older people. Br J Community Nurs. [Internet]. 2017 [acesso em 17 jun 2018]; 22(1). Disponível em: https://doi.org/10.12968/bjcn.2017.22.1.683.

19. Cabrita M de FG, José HMG. The elderly person in the Equipe de Cuidados Continuados Integrados: nursing program for prevention of falls. Revista de Enfermagem UFPE. [Internet]. 2013 [acesso em 15 jun 2018]; 7(1). Disponível em: https://periodicos.ufpe.br/revistas/revistaenfermagem/article/view/10209.

20. Palvanen M, Kannus P, Piirtola M, Niemi S, ParKKari J, Jarvinem M. Effectiveness of the Chaos Falls Clinic in preventing falls and injuries of home-dwelling older adults: A randomised controlled trial. Injury. 
[Internet]. 2014 [acesso em 17 jun 2018]; 45(1). Disponível em: https://doi.org/10.1016/j. injury.2013.03.010.

21. Kamei T, Kajii F, Yamamoto Y, Irie Y, Kozakai R, Sugimoto T, et al. Home modification for fall reduction. JPN Nurs Sci. [Internet]. 2015 [acesso em 17 jun 2018]; 12(3). Disponível em: https://doi.org/10.1111/ jins.12059.

22. Maggi P, Mello J de A, Delye S, Cès S, Macq J, Gosset C, et al Fall determinants and home modifications by occupational therapists to prevent falls: Facteurs déterminants des chutes et modifications du domicile effectuées par les ergothérapeutes pour prévenir les chutes. Can J Occup Ther. [Internet]. 2018 [acesso em 14 jun 2018]; 85(1). Disponível em: https://doi.

org/10.1177/0008417417714284.

23. Wilson N, Kvizhinadze G, Pega F, Nair N, Blakely T. Home modification to reduce falls at a health district level: Modeling health gain, health inequalities and health costs. PLoS One [Internet]. 2017 [acesso em 17 jun 2018]; 12(9). Disponível em: https://journals.plos.org/plosone/article?id=10.1371/journal. pone.0184538.

24. Pega F, Kvizhinadze G, Blakely T, Atkinson J, Wilson N. Home safety assessment and modification to reduce injurious falls in community-dwelling older adults: cost-utility and equity analysis. Inj Prev.

[Internet]. 2016 [acesso em 17 jun 2018]; 22(6). Disponível em: http://dx.doi.org/10.1136/ injuryprev-2016-041999.

25. Ueda T, Higuchi Y, Imaoka M, Todo E, Kitagawa T, Ando S. Tailored education program using home floor plans for falls prevention in discharged older patients: A pilot randomized controlled trial. Arch Gerontol Geriatr. [Internet]. 2017 [acesso em 17 jun 2018]; 71. Disponível em: https://doi.org/10.1016/j. archger.2017.02.010.

26. Rimland JM, Abraha I, Dell'Aquila G, Cruz-Jentoft A, Soiza RL, Gudmundsson A, et al. Nonpharmacological interventions to prevent falls in older patients: Clinical practice recommendations - the SENATOR ONTOP Series. EuGMS. [Internet]. 2017 [acesso em 17 jun 2018]; 8(5-6). Disponível em: https://doi.org/10.1016/j.eurger.2017.07.013.

27. Stark S, Somerville E, Conte J, Keglovits M, Hu YL, Carpenter C, et al. Feasibility Trial of Tailored Home Modifications: Process Outcomes. Am J Occup Ther [Internet]. 2018 [acesso em 16 jun 2018]; 72(1). Disponível em: https://doi.org/10.5014/ajot.2018.021774.

28. Rimland JM, Abraha I, Dell'Aquila G, Cruz-Jentoft A, Soiza R, Gudmusson A, et al. Effectiveness of Non-Pharmacological Interventions to Prevent Falls in Older People: A Systematic Overview. The SENATOR Project ONTOP Series. PLoS ONE. [Internet]. 2016 [acesso em 17 jun 2018]; 11(8). Disponível em: https://doi.org/10.1371/journal.pone.0161579.

29. Hammarlund CS, Hagell P, Westergren A. Fall Risk and Its Associated Factors among Older Adults without Home-Help Services in a Swedish Municipality. J Community Health Nurs [Internet]. 2016 [acesso em 17 jun 2018]; 33(4). Disponível em: https://www.ncbi.nlm.nih.gov/pubmed/27749090.

30. Silva JA, Moreno GHM, Hayakawa LY, Inoue KC, Cuman RKN. Same-level falls in older adults: factors associated with traumatic brain and spinal cord injuries. Cogitare enferm. [Internet]. 2018 [acesso em 10 dez 2019]; 23(4). Disponível em: http://dx.doi.org/10.5380/ce.v23i4.56325.

31. Cunha LFC da, Baixinho CL, Henriques MA. Preventing falls in hospitalized elderly: design and validation of a team intervention. Rev esc enferm USP. [Internet]. 2019 [acesso em 13 dez 2019]; 53:e3479. Disponível em: http://dx.doi.org/10.1590/S1980-220X2018031803479. 
Recebido: $24 / 01 / 2020$

Finalizado: 25/05/2020

Editora associada: Luciana Puchalski Kalinke

Autor Correspondente:

Cristina Lavareda Baixino

Escola Superior de Enfermagem de Lisboa

Av. Egas Moniz 1600-199 - Lisboa, Portugal

E-mail: crbaixinho@esel.pt

Contribuição dos autores:

Contribuições substanciais para a concepção ou desenho do estudo; ou a aquisição, análise ou interpretação de dados do estudo - CLB

Elaboração e revisão crítica do conteúdo intelectual do estudo - CLB

Aprovação da versão final do estudo a ser publicado - CLB

Responsável por todos os aspectos do estudo, assegurando as questões de precisão ou integridade de qualquer parte do estudo - BWS

\section{(c) (1)}

Este obra está licenciado com uma Licença Creative Commons Atribuição 4.0 Internacional. 